\title{
Estimating the effect of the 2005 change in BCG policy in England: a retrospective cohort study, 2000 to 2015
}

Sam Abbott ${ }^{1}$, Hannah Christensen ${ }^{1}$, Nicky J. Welton ${ }^{1}$, Ellen Brooks-Pollock ${ }^{1}$

1. Bristol Medical School: Population Health Sciences, University of Bristol, Bristol, United Kingdom

Correspondence: Sam Abbott (contact@samabbott.co.uk)

Citation style for this article:

Abbott Sam, Christensen Hannah, Welton Nicky)., Brooks-Pollock Ellen. Estimating the effect of the 2005 change in BCG policy in England: a retrospective cohort study, 2000 to 2015. Euro Surveill. 2019;24(49):pii=1900220. https://doi.org/10.2807/1560-7917.ES.2019.24.49.1900220

Background: In 2005 in England, universal Bacillus Calmette-Guérin (BCG) vaccination of school-age children was replaced by targeted BCG vaccination of high-risk neonates. Aim: Estimate the impact of the 2005 change in BCG policy on tuberculosis (TB) incidence rates in England. Methods: We conducted an observational study by combining notifications from the Enhanced Tuberculosis Surveillance system, with demographic data from the Labour Force Survey to construct retrospective cohorts relevant to both the universal and targeted vaccination between 1 January 2000 and 31 December 2010. We then estimated incidence rates over a 5 -year follow-up period and used regression modelling to estimate the impact of the change in policy on TB. Results: In the non-United Kingdom (UK) born, we found evidence for an association between a reduction in incidence rates and the change in BCG policy (school-age incidence rate ratio (IRR): $0.74 ; 95 \%$ credible interval (Crl): 0.61 to 0.88 and neonatal IRR: $0.62 ; 95 \% \mathrm{Crl}: 0.44$ to 0.88 ). We found some evidence that the change in policy was associated with an increase in incidence rates in the UK born school-age population (IRR: $1.08 ; 95 \% C r l: 0.97$ to 1.19) and weaker evidence of an association with a reduction in incidence rates in UK born neonates (IRR: 0.96; $95 \% \mathrm{Crl}: 0.82$ to 1.14$)$. Overall, we found that the change in policy was associated with directly preventing 385 (95\%Crl: -105 to 881 ) cases. Conclusions: Withdrawing universal vaccination at school age and targeting vaccination towards high-risk neonates was associated with reduced incidence of TB. This was largely driven by reductions in the non-UK born with cases increasing in the UK born.

\section{Introduction}

In 2005, England changed its Bacillus Calmette-Guérin (BCG) vaccination policy against tuberculosis (TB) from a universal programme aimed at 13 and 14 yearolds to a targeted programme aimed at high-risk neonates. High-risk babies are identified by local TB incidence and by the parents' and grandparents' country of origin. The change in policy was motivated by evidence of reduced TB transmission [1-3], high effectiveness of the $B C G$ vaccine in young children [4-6] and variable effectiveness in adults [7]. Little work has been done to evaluate the impact of this change in vaccination policy.

Globally, several countries with low TB incidence have moved from universal vaccination, either of those at school age or neonates, to targeted vaccination of neonates considered at high risk of TB [7]. In Sweden, which discontinued universal vaccination of neonates in favour of targeted vaccination of those at high risk, incidence rates in Swedish-born children increased slightly after the change in policy [8]. In France, which also switched from universal vaccination of children to targeted vaccination of those at high risk, a study found that targeted vaccination may have reduced coverage in those most at risk [9].

The number of TB notifications in England increased from 6,929 in 2004 to 8,280 in 2011 but has since declined to 5,137 in 2017 [1]. A recent study found that this reduction may be linked to improved TB interventions [10]. Directly linking trends in TB incidence to transmission is complex because after an initial infection an individual may either develop active disease, or enter a latent stage, which then may later develop into active disease. Incidence in children is a proxy of TB transmission, because any active TB disease in this population is attributable to recent transmission. Using this approach it is thought that TB transmission has been falling in England since 2011, a notion supported by strain typing [1]. Nevertheless, the effect of the change in BCG policy, which is likely to have reduced incidence rates in children, has not been assessed.

Although the long-term effects of BCG vaccination such as reducing the reactivation of latent cases and decreasing onwards transmission are not readily detectable over short time scales, the direct effects of vaccination on incidence rates can be estimated in vaccinated populations, when compared with comparable 


\section{FIGURE}

Mean incidence rates per 100,000 population, with 95\% confidence intervals for each retrospective cohort ${ }^{\mathrm{a}}$ studied to assess the effect of the 2005 change in BCG policy, stratified by the vaccination policy and UK birth status, England, 2000-2015

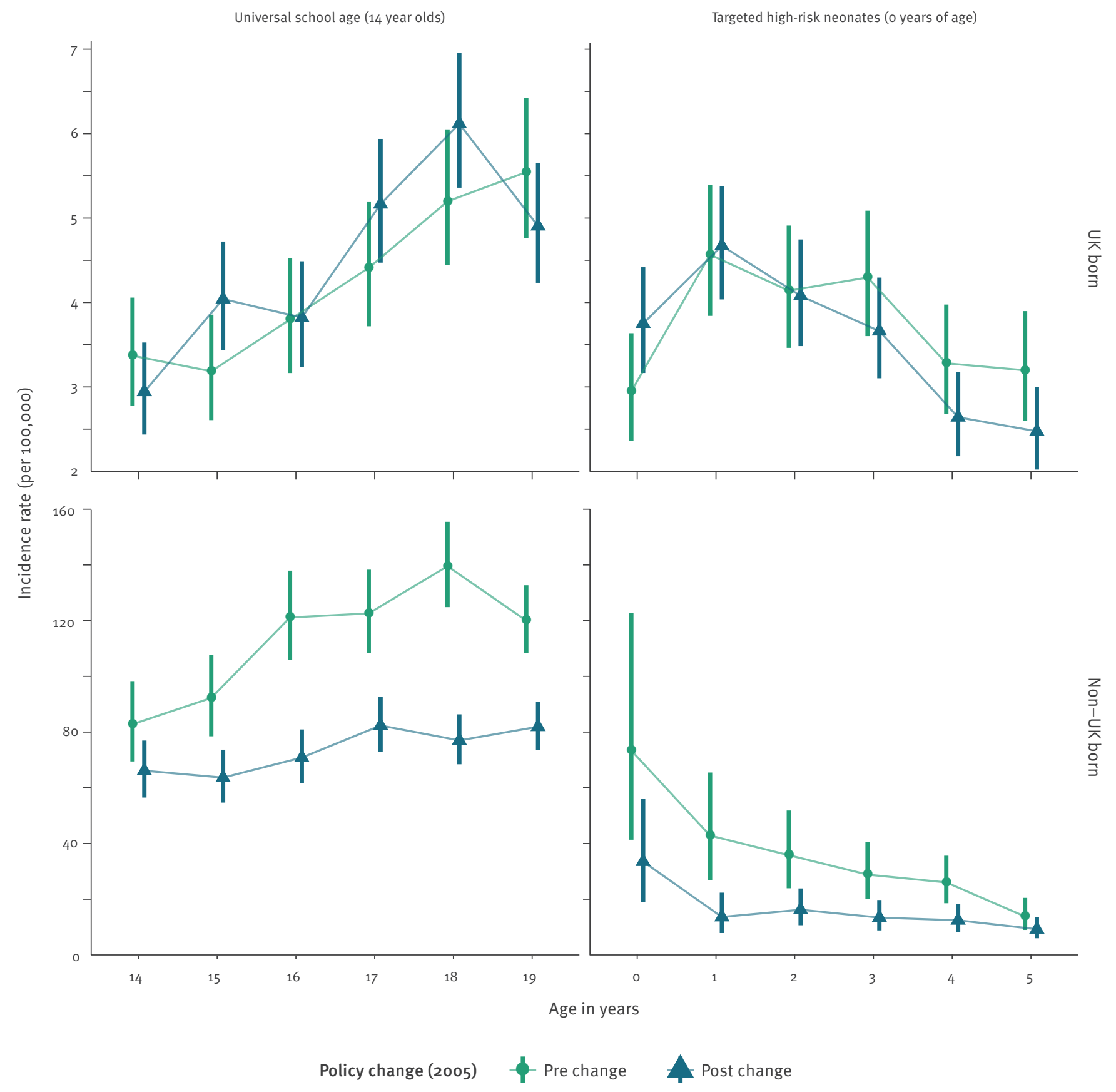

BCG: Bacillus Calmette-Guérin; UK: United Kingdom.

${ }^{\text {a }}$ Cohort definitions figure in Table 1.

The top and bottom panels are on different scales in order to highlight trends in incidence rates over time. 
Summary of relevance and eligibility criteria of cohorts studied to assess the effect of the 2005 change in BCG policy, England, 2000-2010.

\begin{tabular}{|c|c|c|c|c|c|}
\hline Cohort & $\begin{array}{l}\text { Vaccination } \\
\text { programme }\end{array}$ & $\begin{array}{c}\text { Eligible for the } \\
\text { programmea }\end{array}$ & Birth status & $\begin{array}{l}\text { Age in years at } \\
\text { study entry }\end{array}$ & Year of study entry \\
\hline Cohort 1 & Universal & Yes & UK born & 14 & $2000-2004$ \\
\hline Comparison cohort 1 & Universal & No & UK born & 14 & $2005-2010$ \\
\hline Cohort 1 & Universal & Yes & Non-UK born & 14 & $2000-2004$ \\
\hline Comparison cohort 1 & Universal & No & Non-UK born & 14 & $2005-2010$ \\
\hline Cohort 2 & Targeted & Yes & UK born & Birth & $2005-2010$ \\
\hline Comparison cohort 2 & Targeted & No & UK born & Birth & $2000-2004$ \\
\hline Cohort 2 & Targeted & Yes & Non-UK born & Birth & $2005-2010$ \\
\hline Comparison cohort 2 & Targeted & No & Non-UK born & Birth & $2000-2004$ \\
\hline
\end{tabular}

BCG: Bacillus Calmette-Guérin; UK: United Kingdom.

a Eligible signifies that the cohort fit the criteria for the vaccination programme and entered the study during the time period it was in operation not that the cohort was vaccinated by the vaccination programme.

unvaccinated populations [11]. Here, we aimed to estimate the impact of the 2005 change in BCG policy on incidence rates in England, in both the United Kingdom (UK) and non-UK born populations, directly affected by it.

\section{Methods}

\section{Data sources}

Data on all notifications from the Enhanced Tuberculosis Surveillance (ETS) system from 1 January 2000 to 31 December 2015 were obtained from Public Health England (PHE). The ETS is maintained by PHE and contains demographic, clinical and microbiological data on all notified cases in England. A descriptive analysis of TB epidemiology in England is published each year, which fully details data collection and cleaning [1]. Tuberculosis is highly heterogeneous in England with the majority of cases occurring in urban, non-UK born populations. The yearly PHE report contains more descriptive detail [1].

We obtained yearly population estimates from the April to June Labour Force Survey (LFS) for years 2000 to 2015. The LFS is a household study of the employment circumstances of the UK population, which provides the official measures of employment and unemployment in the UK. It is also used to study population demographics such as ethnicity, country of birth and age. Reporting practices have changed with time so the appropriate variables for age, country of origin, country of birth, and survey weight were extracted from each yearly extract, standardised and combined into a single dataset.

\section{Constructing retrospective cohorts}

We constructed retrospective cohorts of TB cases and individuals using the ETS and the LFS. Tuberculosis cases were extracted from the ETS based on date of birth and date of TB notification.
Cohort 1: individuals aged 14 years between 2000 and 2004, who were notified with TB while aged between 14 and 19 years.

Comparison cohort 1: individuals aged 14 years between 2005 and 2010, who were notified with TB while aged between 14 and 19 years.

Cohort 2: individuals born between 2005 and 2010, who were notified with TB while aged o to 5 years.

Comparison cohort 2: individuals born between 2000 and 2004, who were notified with TB while aged o to 5 years.

Cohorts were stratified by vaccination programme using age criteria and then stratified further by whether the scheme was in place during the time period they entered the study. Each cohort was further stratified by UK birth status, with both non-UK born and UK born cases assumed to have been exposed to England's vaccination policy. Corresponding population cohorts were calculated using the LFS population estimates, resulting in eight population level cohorts, each with 5 years of follow-up (Table 1 ).

\section{Statistical methods overview}

We estimated incidence rates (with 95\%confidence intervals (CI)) by year, age and place of birth as (number of cases) divided by (number of individuals of corresponding age). UK birth status was incomplete, with some evidence of a missing not at random mechanism. We imputed the missing data using a gradient boosting method (GBM; see Supplement). We then used a descriptive analysis to describe the observed trends in age-specific incidence rates over the study period, comparing incidence rates in the study populations relevant to both vaccination programmes before and after the change in BCG policy. 
TABLE 2

Incidence rate ratios of tuberculosis in the UK born and non-UK born cohorts relevant to the universal schoolage BCG vaccination scheme, using the best fitting models ${ }^{a}$ as determined by comparison of the LOOIC, England, $2000-2015$

\begin{tabular}{|c|c|c|}
\hline \multirow{2}{*}{ Variable } & \multicolumn{2}{|c|}{ IRR $(95 \% \mathrm{Crl})$} \\
\hline & UK born & Non-UK born \\
\hline \multicolumn{3}{|l|}{ Policy change ${ }^{b}$} \\
\hline Post-change & $1.08(0.97$ to 1.19$)$ & $0.74(0.61$ to 0.88$)$ \\
\hline \multicolumn{3}{|l|}{ Age in years } \\
\hline 14 & Reference & Reference \\
\hline 15 & $1.18(0.98$ to 1.42$)$ & $1.03(0.87$ to 1.22$)$ \\
\hline 16 & $1.24(1.03$ to 1.50$)$ & $1.25(1.07$ to 1.47$)$ \\
\hline 17 & 1.59 (1.33 to 1.91) & $1.40(1.19$ to 1.63$)$ \\
\hline 18 & $1.92(1.60$ to 2.30$)$ & $1.47(1.26$ to 1.73$)$ \\
\hline 19 & $1.80(1.49$ to 2.17$)$ & $1.47(1.24$ to 1.73$)$ \\
\hline \multicolumn{3}{|l|}{ Incidence rate } \\
\hline Non-UK born incidence rate (per standard deviation) & NA & 1.11 (1.03 to 1.19) \\
\hline \multicolumn{3}{|l|}{ Year of study eligibility, group level } \\
\hline Intercept (standard deviation) & NA & $1.13(1.05$ to 1.26$)$ \\
\hline \multicolumn{3}{|l|}{ Year of study eligibility, individual level } \\
\hline 2000 & NA & $1.10(0.96$ to 1.29$)$ \\
\hline 2001 & NA & $1.06(0.93$ to 1.24$)$ \\
\hline 2002 & NA & $1.07(0.94$ to 1.25$)$ \\
\hline 2003 & NA & $0.90(0.76$ to 1.03$)$ \\
\hline 2004 & NA & 0.89 (0.75 to 1.02) \\
\hline 2005 & NA & $0.98(0.85$ to 1.12$)$ \\
\hline 2006 & NA & 1.13 (0.99 to 1.33) \\
\hline 2007 & NA & $1.04(0.91$ to 1.20$)$ \\
\hline 2009 & NA & 0.95 (0.81 to 1.08) \\
\hline 2010 & NA & 0.96 (o.82 to 1.11$)$ \\
\hline
\end{tabular}

BCG: Bacillus Calmette-Guérin; Crl: credible interval; IRR: incidence rate ratio; LOOIC: leave one out cross validation information criterion; SE: standard error; NA: not applicable (i.e. model terms were not included in the given cohort); UK: United Kingdom.

a The best fitting model for the UK born was a negative binomial model adjusting with fixed effects for the change in policy, age and incidence rates in the UK born (Model7; Supplement Table S1). For the non-UK born the best fitting model was a negative binomial model with a random intercept for year of study entry, adjusting with fixed effects for the change in policy, age and incidence rates in the non-UK born (Model 17; Supplement Table S1).

b There was an improvement in the LOOIC score of 0.52 (SE: 2.63) from dropping the change in policy from the model in the UK born cohort and a -3.02 (SE: 3.52) improvement in the non-UK born cohort.

We calculated incidence rate ratios (IRRs) with 95\% credible intervals (Crls) for the change in incidence rates associated with the change in BCG vaccination policy (modelled as a binary breakpoint at the start of 2005) for both the UK born and non-UK born populations that were relevant for the universal programme and for the targeted programme, using a range of models. We considered the following covariates: age $[1,7]$, incidence rates in both the UK born and non-UK born who were not in the age group of interest [1] and year of study entry (as a random intercept). We evaluated a range of models using a statistically rigorous criterion that accounted for model fit and complexity, for model selection.

\section{Statistical modelling details}

We first investigated a univariable Poisson model, followed by combinations of covariates (Supplement Table $\mathrm{S}_{1}$ ). We also investigated a negative binomial model, adjusting for the same covariates as in the best fitting Poisson model. The models were estimated with a Bayesian approach using Markov Chain Monte Carlo (MCMC) with default weakly informative priors (Supplement). Model fit, penalised by model complexity, was assessed using the leave one out cross validation information criterion (LOOIC) and its standard error [12]. Models were ranked by goodness of fit, using their LOOIC, with a smaller LOOIC indicating a better fit to the data after adjusting for the complexity of the 


\section{TABLE 3}

Incidence rate ratios of tuberculosis, in the UK born and non-UK born cohorts relevant to the targeted neonatal BCG vaccination scheme, using the best fitting models ${ }^{\mathrm{a}}$ as determined by comparison of the LOOIC, England, 2000-2015

\begin{tabular}{|c|c|c|}
\hline \multirow{2}{*}{ Variable } & \multicolumn{2}{|c|}{ IRR $(95 \% \mathrm{Crl})$} \\
\hline & UK born & Non-UK born \\
\hline \multicolumn{3}{|l|}{ Policy change ${ }^{b}$} \\
\hline Post-change & $0.96(0.82$ to 1.14) & $0.62(0.44$ to 0.88$)$ \\
\hline \multicolumn{3}{|l|}{ Age } \\
\hline 0 & Reference & Reference \\
\hline 1 & $1.39(1.20$ to 1.61$)$ & 0.49 (0.30 to 0.83$)$ \\
\hline 2 & $1.24(1.06$ to 1.44$)$ & 0.49 (0.30 to 0.80$)$ \\
\hline 3 & 1.21 (1.03 to 1.41$)$ & $0.42(0.26$ to 0.68$)$ \\
\hline 4 & $0.90(0.76$ to 1.06$)$ & 0.41 (0.25 to 0.66$)$ \\
\hline 5 & $0.89(0.75$ to 1.06$)$ & 0.27 (0.16 to 0.45$)$ \\
\hline \multicolumn{3}{|l|}{ Incidence } \\
\hline Non-UK born incidence rate (per standard deviation) & NA & $1.25(1.04$ to 1.51$)$ \\
\hline \multicolumn{3}{|l|}{ Year of study eligibility, group level } \\
\hline Intercept (standard deviation) & $1.13(1.04$ to 1.26$)$ & NA \\
\hline \multicolumn{3}{|l|}{ Year of study eligibility, individual level } \\
\hline 2000 & 0.83 (0.68 to 0.99) & NA \\
\hline 2001 & $0.93(0.79$ to 1.07$)$ & NA \\
\hline 2002 & $1.08(0.95$ to 1.28$)$ & NA \\
\hline 2003 & 1.07 (0.93 to 1.26) & NA \\
\hline 2004 & $1.12(0.97$ to 1.32$)$ & NA \\
\hline 2005 & 1.02 (0.89 to 1.17) & NA \\
\hline 2006 & 1.02 (0.89 to 1.17) & NA \\
\hline 2007 & 0.97 (0.83 to 1.11) & $\mathrm{NA}$ \\
\hline 2010 & 0.98 (0.85 to 1.13) & NA \\
\hline
\end{tabular}

BCG: Bacillus Calmette-Guérin; Crl: credible interval; IRR: incidence rate ratio; LOOIC: leave one out cross validation information criterion; SE: standard error; NA: not applicable (i.e. model terms were not included in the given cohort); UK: United Kingdom.

${ }^{a}$ The best fitting model for the UK born was a Poisson model with a random intercept for year of study entry, adjusting with fixed effects for the change in policy, age and incidence rates in the UK born (Model 16; Supplement Table S1). For the non-UK born a negative binomial model was the best fit, adjusting with fixed effects for the change in policy, age and incidence rates in the non-UK born (Model 8; Supplement Table S1).

b There was an improvement in the LOOIC score of 0.92 (SE: 1.07) from dropping the change in policy from the model in the UK born cohort and a -3.45 (SE: 4.63) improvement in the non-UK born cohort.

model. No formal threshold for a change in the LOOIC was used, with changes in the LOOIC being evaluated in the context of their standard error (SE).

The inclusion of the change in policy in the best fitting model was tested by refitting the model excluding the change in policy and estimating the improvement in the LOOIC.

Once the best fitting model had been identified we estimated the number of cases prevented, from 2005 until $\mathbf{2 0 1 5}$, for each vaccination programme in the study population relevant to that programme (Supplement).

\section{Implementation}

R 3.5.2 was used for all analyses [13]. Missing data imputation using a GBM was implemented using the h20 package [14]. Incidence rates, with $95 \% \mathrm{Cls}$, were calculated using the epiR package [15]. The brms package [16] and STAN [17] were used to perform MCMC. Models were run until convergence (four chains with a burn in of 10,000 and 10,000 sampled iterations each), with convergence being assessed using trace plots and the $R$ hat diagnostic [17]. All numeric confounders were centred and scaled by their standard deviation and age was adjusted for using single year of age categories.

\section{Ethical statement}

Ethical approval was not required for this study, which used anonymised secondary data sources only. 
TABLE 4

Estimated number of cases prevented, from 2005 until 2015, for each vaccination programme in the study population relevant to that programme, using the best fitting model for each cohort, England, 2000-2015

\begin{tabular}{|c|c|c|c|}
\hline Vaccination programme & Birth status & Cases prevented $(95 \% \mathrm{Crl})$ & Notified cases \\
\hline \multirow{3}{*}{ 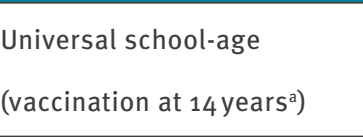 } & All & $-291(24$ to -571$)$ & 2,364 \\
\hline & UK born & $76(188$ to -26$)$ & 969 \\
\hline & Non-UK born & $-367(-165$ to -546$)$ & 1,395 \\
\hline \multirow{3}{*}{$\begin{array}{l}\text { Targeted high-risk neonates } \\
\text { (vaccination at birth) }\end{array}$} & All & $94(-81$ to 310$)$ & 906 \\
\hline & UK born & $30(-95$ to 173) & 800 \\
\hline & Non-UK born & 65 (14 to 137) & 106 \\
\hline \multirow{3}{*}{ Change in policyb } & All & $385(-105$ to 881$)$ & 3,270 \\
\hline & UK born & $-46(-284$ to 199$)$ & 1,769 \\
\hline & Non-UK born & 431 (179 to 682) & 1,501 \\
\hline
\end{tabular}

Crl: credible interval; UK: United Kingdom.

a In effect, vaccination was implemented across a school year (hence in 13 and 14 year olds) but for the synthetic cohort used in this study, vaccination at 14 years of age was used as an entry criterion.

${ }^{b}$ Estimated total number of cases prevented due to the change in vaccination policy in 2005 .

\section{Results}

\section{Descriptive analysis}

During the study period, there were 114,820 notifications of TB in England, of which 93\% $(106,765 / 114,820)$ had their birth status recorded. Of notifications with a known birth status $27 \%(29,096 / 106,765)$ were UK born, while among notification with an imputed birth status, $33 \%(2,634 / 8,055)$ were UK born. Trends in incidence rates varied by age group and UK birth status (Supplement). During the study period, there were 1,729 UK born cases and 2,797 non-UK born cases in individuals relevant to the universal school scheme and 1,431 UK born cases and 238 non-UK born cases relevant to the targeted neonatal scheme, who fit our age criteria. Univariable evidence for differences between mean incidence rates before and after the change in BCG policy in the UK born was weak. In the non-UK born incidence rates were lower after the change in BCG policy in both the cohort relevant to the universal school-age scheme and the cohort relevant to the targeted neonatal scheme (Figure).

\section{Adjusted estimates of the effects of the change} in policy on school-age children

In the UK born cohort relevant to universal vaccination there was some evidence across all models adjusting for age, that ending the scheme was associated with a modest increase in TB rates (Supplement Table S2). Using the LOOIC goodness of fit criteria, the best fitting model was found to be a negative binomial model that adjusted for the change in policy, age and incidence rates in the UK born (Table 2). In this model there was some evidence of an association between the change in policy and an increase in incidence rates in those at school age who were UK born, with an IRR of 1.08 (95\% Crl: 0.97 to 1.19). Dropping the change in policy from the model resulted in a small decrease in the LOOIC (0.52; SE: 2.63) but the change was too small, with too large a SE, to conclusively state that excluding the change in policy from the model improved the quality of model fit. We found that it was important to adjust for UK born incidence rates, otherwise the impact from the change in BCG vaccination policy was overestimated.

For the comparable non-UK born cohort who were relevant to the universal vaccination there was evidence, in the best fitting model, that ending the scheme was associated with a decrease in incidence rates (IRR: 0.74 ; 95\% Crl: 0.61 to 0.88). The best fitting model was a negative binomial model, which adjusted for the change in policy, age, incidence rates in the non-UK born and year of eligibility as a random effect (Table 2). We found omitting change in policy from the model resulted in poorer model fit (LOOIC increase of 3.02; $\mathrm{SE}: 3.52)$, suggesting that the policy change was an important factor explaining changes in incidence rates, after adjusting for other covariates.

All models that adjusted for incidence rates in the UK born or non-UK born estimated similar IRRS (Supplement Table S3).

Adjusted estimates of the effect of the change in policy in those relevant to the targeted neonatal programme

For the UK born cohort relevant to the targeted neonatal vaccination programme, the evidence of an association, across all models, was mixed and Crls were wide compared with models for the UK born cohort relevant to the universal school-age vaccination programme (Supplement Table S4). The best fitting model was a Poisson model, which adjusted for the change in policy, age, UK born incidence rates and year of study entry with a random effect (Table 3). In this model, there was weak evidence of an association between the change in BCG policy and a decrease in incidence rates in UK born neonates, with an IRR of 0.96 ( $95 \% \mathrm{Crl}: 0.82$ to 1.14 ). 
There was weak evidence to suggest that dropping the change in policy from this model improved the quality of the fit, with an improvement in the LOOIC score of 0.92 (SE: 1.07). This suggests that the change in policy was not an important factor for explaining incidence rates, after adjusting for covariates. Models, which also adjusted for non-UK born incidence rates estimated that the change in policy was associated with no change in incidence rates in the relevant cohort of neonates (Supplement).

For the comparable non-UK born cohort who was relevant to the targeted neonatal vaccination programme there was evidence, across all models, that change in policy was associated with a large decrease in incidence rates (IRR: $0.62 ; 95 \% \mathrm{Crl}: 0.44$ to 0.88 ) in the best fitting model (Table 3 ). The best fitting model was a negative binomial model that adjusted for the change in policy, age, and non-UK born incidence rates (Table 3). All models for this cohort, which at least adjusted for age, estimated comparable effects of the change in policy (Supplement Table S5).

Magnitude of the estimated impact of the change in Bacillus Calmette-Guérin policy We estimate that the change in vaccination policy was associated with preventing 385 (95\%Crl: -105 to 881 ) cases from 2005 until the end of the study period in the directly impacted populations after 5 years of follow-up (Table 4). The majority of the cases prevented were in the non-UK born, with cases increasing slightly overall in the UK born. This was due to cases increasing in the UK born at school age despite a decrease in UK born neonates, although both these estimates had large Crls.

\section{Discussion}

In the non-UK born we found evidence of an association between the change in BCG policy and a decrease in TB incidence rates in both those at school age and neonates, after 5 years of follow-up. We found some evidence that the change in BCG policy was associated with a modest increase in incidence rates in the UK born population who were relevant to the universal schoolage scheme and weaker evidence of a small decrease in incidence rates in the UK born population relevant to the targeted neonatal scheme. Overall, we found that the change in policy was associated with preventing 385 (95\% Crl: -105 to 881) cases in the study population, from 2005 until the end of the study period, with the majority of the cases prevented in the non-UK born.

We were unable to estimate the impact of the change in BCG policy after 5 years post vaccination, so both our estimates of the positive and negative consequences are likely to be underestimates of the ongoing impact. Tuberculosis is a complex disease and the BCG vaccine is known to offer imperfect protection, which has been shown to vary both spatially and with time since vaccination $[18,19]$. By focusing on the impact of the change in policy on the directly affected populations within a short period of time and by employing a multi-model approach we have limited the potential impact of these issues. Our study was based on a routine observational dataset (ETS), and a repeated survey (LFS) both of which may have introduced bias. While the LFS is a robust data source, widely used in academic studies [20-22], it is susceptible to sampling errors particularly in the young and in the old, which may have biased the estimated incidence rates. As the ETS is a routine surveillance system some level of missing data are inevitable. However, the UK birth status was relatively complete (93\%) and we imputed missing values using an approach, which accounted for missing not at random mechanisms captured by variables included in the imputation model. We were unable to adjust for known demographic risk factors for TB, notably socioeconomic status [23,24] and ethnicity [23-25]. However, this confounding is likely to be mitigated by our use of multiple cohorts and our adjustment for incidence rates in the UK born and non-UK born. Finally, we have assumed that the effect we have estimated for the change in BCG policy is due to the changes in BCG vaccination policy as well as other associated changes in TB control policy, after adjusting for hypothesised confounders. However, there may have been additional policy changes, which we have not accounted for.

While little work has been done to assess the impact of the 2005 change in BCG vaccination, several other studies have estimated the impact of changing BCG vaccination policy, although typically only from universal vaccination of neonates to targeted vaccination of high-risk neonates. A previous study in Sweden found that incidence rates in Swedish-born children increased after high-risk neonatal vaccination was implemented in place of a universal neonatal programme. This corresponds with our finding that introducing neonatal vaccination had little impact on incidence rates in UK born neonates. Theoretical approaches have indicated that targeted vaccination of those at high risk may be optimal in low incidence settings [26]. Our study extends this work by also considering the age of those given $B C G$ vaccination, although we were unable to estimate the impact of a universal neonatal scheme as this has never been implemented nationally in England. It has previously been shown that targeted vaccination programmes may not reach those considered most at risk [27]. Our findings may support this view as we observed only a small decrease in incidence rates in UK born neonates after the introduction of the targeted neonatal vaccination programme. Alternatively, the effectiveness of the BCG in neonates, in England, may be lower than previously thought as we only observed a small decrease in incidence rates, while a previous study estimated BCG coverage at 68\% (95\% Cl:65\% to $71 \%$ ) among those eligible for the targeted neonatal vaccination programme [28].

This study indicates that the change in England's BCG vaccination policy was associated with a modest increase in incidence in the UK born who were relevant 
to the school-age vaccination programme, and with a small reduction in incidence in the UK born who were relevant to the high-risk neonatal vaccination programme, although both these estimates had wide Crls. We found stronger evidence of an association between the change in policy and a decrease in incidence rates in the non-UK born populations relevant to both programmes. This suggests that the change of vaccination policy to target high-risk neonates may have resulted in an increased focus on high-risk non-UK born individuals who may not have been the direct targets of the vaccination programme. Further validation is required using alternative study designs, but this result should be taken into account when vaccination policy changes are being considered. Our results should be interpreted carefully, especially in the non-UK born, as we could not fully rule out the impact of other TB control measures that may have been changed at the same time as vaccination policy. The severity of TB is known to differ across age groups, with children having a higher incidence of TB meningitis, which can be severe, compared with other age groups [1]. This variation should also be considered when evaluating these results.

It is well established that interventions against infectious diseases, such as TB, should be evaluated not only for their direct effects but also for future indirect effects via ongoing transmission. Statistical approaches such as those used in this paper are not appropriate for capturing these future indirect effects, and instead dynamic disease models should be used. In addition, this study could not evaluate the impact of the neonatal programme on the high-risk population it targets, due to a lack of reliable data. Improved coverage data for the BCG programme is required to more fully evaluate its ongoing impact. As only 5 years of follow-up data were available - and BCG vaccination has been shown to provide long lasting protection in the UK - repeating this study once more data are available may alter the findings [29]. For this reason, this study has been implemented with reproducibility in mind - please see the code reference below for details. Finally, the results from this study could be combined with estimates of the impact of TB disease, stratified by age, to give an estimate of the overall impact of the change in policy that accounts for the severity of disease.

Accessibility of data and programming code

The code used to clean the data used in this paper can be found at: DOI: 10.5281/zenodo.2551555.

The code for the analysis contained in this paper can be found at: DOI: 10.5281/zenodo.2583056.

\section{Acknowledgements}

The authors thank the TB section at Public Health England (PHE) for maintaining the Enhanced Tuberculosis Surveillance
(ETS) system; all the healthcare workers involved in data collection for the ETS.

Funding: SA, HC, EBP and NJW are funded by the National Institute for Health Research Health Protection Research Unit (NIHR HPRU) in Evaluation of Interventions at University of Bristol in partnership with Public Health England (PHE).

The views expressed are those of the author(s) and not necessarily those of the NHS, the NIHR, the Department of Health or Public Health England.

\section{Conflict of interest}

$\mathrm{HC}$ reports receiving honoraria from Sanofi Pasteur, and consultancy fees from AstraZeneca, GSK and IMS Health, all paid to her employer.

\section{Authors' contributions}

SA, HC, and EBP conceived and designed the work. NJW provided guidance on the statistical methods used. SA undertook the analysis with advice from all other authors. All authors contributed to the interpretation of the data. SA wrote the first draft of the paper and all authors contributed to subsequent drafts. All authors approve the work for publication and agree to be accountable for the work.

\section{References}

1. PHE. Tuberculosis in England 2016 Report (presenting data to end of 2015). Public Heal Engl 2016; Version 1.:173.

2. Fine $P$. Stopping routine vaccination for tuberculosis in schools. BMJ. 2005;331(7518):647-8. https://doi.org/10.1136/ bmj.331.7518.647 PMID: 16179677

3. Teo SSS, Shingadia DV. Does BCG have a role in tuberculosis control and prevention in the United Kingdom? Arch Dis Child. 2006;91(6):529-31. https://doi.org/10.1136/adc.2005.085043 PMID: 16714729

4. Rodrigues LC, Diwan VK, Wheeler JG. Protective effect of BCG against tuberculous meningitis and miliary tuberculosis: a meta-analysis. Int J Epidemiol. 1993;22(6):1154-8. https://doi. org/10.1093/ije/22.6.1154 PMID: 8144299

5. Colditz GA, Brewer TF, Berkey CS, Wilson ME, Burdick E, Fineberg HV, et al. Efficacy of BCG vaccine in the prevention of tuberculosis. Meta-analysis of the published literature. JAMA. 1994:271(9):698-702. https://doi.org/10.1001/ jama.1994.03510330076038 PMID: 8309034

6. Roy A, Eisenhut M, Harris RJ, Rodrigues LC, Sridhar S, Habermann S, et al. Effect of BCG vaccination against Mycobacterium tuberculosis infection in children: systematic review and meta-analysis. BMJ. 2014;349(aug04 5):94643-3. https://doi.org/10.1136/bmj.g4643 PMID: 25097193

7. Zwerling A, Behr MA, Verma A, Brewer TF, Menzies D, Pai M. The BCG World Atlas: a database of global BCG vaccination policies and practices. PLoS Med. 2011;8(3):e1001012. https:// doi.org/10.1371/journal.pmed.1001012 PMID: 21445325

8. Romanus V, Svensson A, Hallander HO. The impact of changing BCG coverage on tuberculosis incidence in Swedish-born children between 1969 and 1989. Tuber Lung Dis. 1992;73(3):150-61. https://doi.org/10.1016/09628479(92)90149-E PMID: 1421348

9. Guthmann JP, Antoine D, Fonteneau L, Che D, Lévy-Bruhl $D$. Assessing $B C G$ vaccination coverage and incidence of paediatric tuberculosis following two major changes in BCG vaccination policy in France. Euro Surveill. 2011;16(12):1-6. PMID: 21457685

10. Thomas HL, Harris RJ, Muzyamba MC, Davidson JA, Lalor MK, Campbell CNJ, et al. Reduction in tuberculosis incidence in the UK from 2011 to 2015: a population-based study. Thorax. 2018;73(8):769-75. https://doi.org/10.1136/ thoraxjnl-2017-211074 PMID: 29674389

11. Parikh SR, Andrews NJ, Beebeejaun K, Campbell H, Ribeiro S, Ward $C$, et al. Effectiveness and impact of a reduced infant schedule of 4 CMen $B$ vaccine against group $B$ meningococcal disease in England: a national observational cohort study. 
Lancet. 2016;388(10061):2775-82. https://doi.org/10.1016/ S0140-6736(16)31921-3 PMID: 28100432

12. Vehtari A, Gelman A, Gabry J. Practical Bayesian model evaluation using leave-one-out cross-validation and WAIC. Stat Comput. 2017;27(5):1413-32. https://doi.org/10.1007/ S11222-016-9696-4

13. R Core Team. R: A Language and Environment for Statistical Computing. 2016. Available from: https://www.r-project.org/

14. $\mathrm{H}_{2} \mathrm{O}$.ai. R Interface for $\mathrm{H}_{2} \mathrm{O}$. 2018. Available from: https:// github.com/h20ai/h20-3

15. Stevenson M, Nunes T, Heuer C, et al. epiR: Tools for the Analysis of Epidemiological Data. 2017. Available from: https:// cran.r-project.org/package $=$ epiR

16. Bürkner P-C. brms: An R Package for Bayesian Multilevel Models using Stan. J Stat Softw. 2017;80(1):1-28. https://doi. org/10.18637/jss.vo80.io1

17. Stan Development Team. $\{R S t a n\}$ : the $\{R\}$ interface to $\{$ Stan $\}$. 2016. Available from: http://mc-stan.org/

18. Mangtani P, Abubakar I, Ariti C, Beynon R, Pimpin L, Fine PE, et al. Protection by BCG vaccine against tuberculosis: a systematic review of randomized controlled trials. Clin Infect Dis. 2014;58(4):470-80. https://doi.org/10.1093/cid/cit790 PMID: 24336911

19. Abubakar I, Pimpin L, Ariti C, Beynon R, Mangtani P, Sterne JA, et al. Systematic review and meta-analysis of the current evidence on the duration of protection by bacillus CalmetteGuérin vaccination against tuberculosis. Health Technol Assess. 2013;17(37):1-372, v-vi. https://doi.org/10.3310/ hta17370 PMID: 24021245

20. French CE, Antoine D, Gelb D, Jones JA, Gilbert RL, Watson $J M$. Tuberculosis in non-UK-born persons, England and Wales, 2001-2003. Int J Tuberc Lung Dis. 2007;11(5):577-84. PMID: 17439685

21. Davies R, Jones M, Lloyd-Williams H. Age and Work-Related Health: Insights from the UK Labour Force Survey. Br J Ind Relat. 2016;54(1):136-59. https://doi.org/10.1111/bjir.12059

22. Lindley J. The over-education of UK immigrants and minority ethnic groups: Evidence from the Labour Force Survey. Econ Educ Rev. 2009;28(1):80-9. https://doi.org/10.1016/j. econedurev.2007.11.003

23. Parslow R, El-Shimy NA, Cundall DB, McKinney PA. Tuberculosis, deprivation, and ethnicity in Leeds, UK, 1982-1997. Arch Dis Child. 2001;84(2):109-13. https://doi. org/10.1136/adc.84.2.109 PMID: 11159282

24. Bhatti N, Law MR, Morris JK, Halliday R, Moore-Gillon J. Increasing incidence of tuberculosis in England and Wales: a study of the likely causes. BMJ. 1995;310(6985):967-9. https:// doi.org/10.1136/bmj.310.6985.967 PMID: 7728031

25. Abubakar I, Laundy MT, French CE, Shingadia D. Epidemiology and treatment outcome of childhood tuberculosis in England and Wales: 1999-2006. Arch Dis Child. 2008;93(12):1017-21. https://doi.org/10.1136/adc.2008.139543 PMID: 18562450

26. Manissero D, Lopalco PL, Levy-Bruhl D, Ciofi Degli Atti ML, Giesecke J. Assessing the impact of different BCG vaccination strategies on severe childhood TB in low-intermediate prevalence settings. Vaccine. 2008;26(18):2253-9. https://doi. org/10.1016/j.vaccine.2008.02.038 PMID: 18400344

27. Feiring B, Laake I, Molden T, Håberg SE, Nøkleby H, Seterelv $\mathrm{SS}$, et al. Do selective immunisation against tuberculosis and hepatitis $B$ reach the targeted populations? A nationwide register-based study evaluating the recommendations in the Norwegian Childhood Immunisation Programme. Vaccine. 2016;34(17):2015-20. https://doi.org/10.1016/j. vaccine.2016.02.060 PMID: 26947498

28. Nguipdop-Djomo P, Mangtani P, Pedrazzoli D, Rodrigues LC Abubakar I. Uptake of neonatal BCG vaccination in England: performance of the current policy recommendations Thorax. 2014;69(1):87-9. https://doi.org/10.1136/ thoraxjnl-2013-203931 PMID: 23853248

29. Nguipdop-Djomo P, Heldal E, Rodrigues LC, Abubakar I, Mangtani P. Duration of BCG protection against tuberculosis and change in effectiveness with time since vaccination in Norway: a retrospective population-based cohort study. Lancet Infect Dis. 2016;16(2):219-26. https://doi.org/10.1016/S14733099(15)00400-4 PMID: 26603173

\section{License, supplementary material and copyright}

This is an open-access article distributed under the terms of the Creative Commons Attribution (CC BY 4.0) Licence. You may share and adapt the material, but must give appropriate credit to the source, provide a link to the licence and indicate if changes were made.

Any supplementary material referenced in the article can be found in the online version.

This article is copyright of the authors or their affiliated institutions, 2019. 